\title{
Review of: "Emergent synchronous beating behavior in spontaneous beating cardiomyocyte clusters"
}

\author{
Sara Pahlavan
}

Potential competing interests: The author(s) declared that no potential competing interests exist.

The paper entitled "Emergent synchronous beating behavior in spontaneous beating cardiomyocyte clusters" has a very well designed experimental procedure to address a fundamental question regarding beating initiation and propagation in the heart. The results did not support the previous hypothesis of "overdrive suppression" and "overdrive by stable inter-beating intervals (IBI)". But they concluded that there might be a complex process governing beating initiation such as "community effect". They used all available in vitro systems i.e. isolated mouse cardiomyocytes and stem cell-derived cardiomyocytes to test their hypothesis. And they also made clusters of cardiomyocytes which resembles cardiac micro-tissue and can be used to investigate synchronization in tissue.

As they indicated in their discussion, it is imperative to use co-culture of different cell types of myocardium. This would result in a more similar situation to in vivo.

And I recommend to use other human pluripotent stem cell (hPSC) lines to check the reproducibility of their results. Because different hPSC lines result in cardiomyocytes with slightly different electrophysiological properties. Furthermore, it would be useful to compare cardiomyocytes generated from different differentiation protocols for further validation. 